\title{
BMENTORÍA: EXPERIENCIA PARA LOS ESTUDIANTES NUEVOS EN UNA UNIVERSIDAD A DISTANCIA
}

\author{
BMENTORING: AN EXPERIENCE FOR NEW STUDENTS AT DISTANCE \\ UNIVERSITY
}

\author{
María José Corral Carrillo ${ }^{1}$ \\ Ana María Martín Cuadrado \\ Universidad Nacional de Educación a Distancia, España.
}

\section{RESUMEN}

Los planes de orientación y acogida surgen en la universidad como una de las medidas facilitadoras en las situaciones de tránsito que sobrevienen en esta etapa educativa. Una estrategia reconocida como medida muy recomendable para acompañar a los estudiantes en su primer año de universidad es la mentoría entre iguales. Este artículo pretende, por un lado, ofrecer una panorámica sobre el programa piloto de bMentoría llevado a cabo en el Centro Asociado de la UNED en Sevilla iniciado en el curso académico 2014/2015; y por otro, presentar algunos de los resultados del primer año. La metodología se ha realizado mediante un estudio exploratorio, de carácter cuantitativo, descriptivo e inferencial que incorpora un análisis de conglomerados a fin de detectar grupos o perfiles en función de las dificultades encontradas al inicio de sus estudios. El tipo de necesidades detectadas corroboran los datos obtenidos en otras investigaciones (Callejo, 2001; García-Aretio, 1994; Sánchez-Elvira, Fernández y Amor, 2006; Sánchez, Manzano-Soto, Martín-Cuadrado, Oliveros, Rísquez, y Suárez, 2009), aunque despuntan otras necesidades relacionadas con las competencias digitales que les impide realizarse personalmente y participar

${ }^{1}$ Correspondencia: María José Corral Carrillo. Escuela Internacional de Doctorado de la UNED. Facultad de Educación. C/ Juan del Rosal, 14. 28040, Madrid. 
en la comunidad educativa. Los medios electrónicos más usados en la relación de bMentoría son el WhatsApp y el e-correo, con un porcentaje de respuesta del $56 \%$ y el $40 \%$, respectivamente. Los temas importantes se resuelven en el centro asociado, preferiblemente. El modelo de bMentoría muestra la importancia de combinar espacios formales e informales, presenciales y virtuales en la relación entre iguales.

Palabras clave: Educación superior, bmentoría, orientación universitaria, blearning, estudiantes de nuevo ingreso

\begin{abstract}
Orientation and reception plans emerge in universities as one of the facilitating measures in transit situations arising in this educative stage. A strategy recognized as a highly recommended measure to accompany the students in their first year at university, is peer mentoring. This article intends, on the one hand, to offer an overview of the pilot program of bMentorship carried out in the Associated Center of the UNED (Spanish Open University) in Seville started during the 2014/2015 academic year; and on the other hand. The methodoly have been supported through exploratory study, as well as quantitative, descriptive and inferential, in the dame way It implement cluster analysis techniques in order to detect either groups or profiles according to the difficulties encountered at the start of their degrees. The type of detected needs corroborates the data obtained in other similar researches (Callejo, 2001; García-Aretio 1994; Sánchez-Elvira, Fernández and Amor, 2006; Sánchez, Manzano-Soto, Martín-Cuadrado, Oliveros, Rísquez, and Suárez, 2009), although other needs related to digital skills preventing them from personal development and participation in the educational community stand out. The most used social media in bMentoría's for communication are WhatsApp and email, with a response rate of $56 \%$ and $40 \%$, respectively. The resolution of important issues is performed in the context of the associated center, preferably. The bMentoría model shows the importance of combining formal and informal spaces, both face-to-face and virtual, in the development of the relationship between peers.
\end{abstract}

Key Words: Higher education, mentoring, university orientation, b-learning, Incoming students

\title{
Introducción
}

La mentoría es un procedimiento de orientación y acompañamiento utilizado desde épocas muy antiguas. Calderón y Corts (1995) comentan que griegos y persas lo utilizaban como estrategia de enseñanza. Juan de la Cuesta, en 1589, utiliza la mentoría como una estrategia didáctica para la mejora del rendimiento (Galino, 1968). En 1801, Joseph Lancaster empezó a acuñar el término "Mentoría" en las escuelas, utilizando el sistema de "enseñanza mutua" (Lancaster, 1803). Finkelsztein, en 1994, redescubre el término y empieza a utilizar en diferentes contextos educativos (Finkelsztein, 1994).

A lo largo del tiempo, se ha podido comprobar que la mentoría es una estrategia educativa que pretende ir más allá del asesoramiento en actividades concretas. Sundli (2007) proporciona un enfoque de mentoría muy apropiado:

"La mentoría es guiar y apoyar al aprendiz para facilitarle las transiciones difíciles; mucho más en una universidad a distancia, es suavizar el camino, para aprovechar los 
recursos y reconocer las oportunidades que le ofrece; capacitarle, alentarle, así como indicarle, dirigirle e instruirle. La mentoría tiene que ver con desarrollo personal y profesional continuos, en el proceso, los valores personales y profesionales se someten a escrutinio y son materia de cambio" (p. 205).

Casado-Muñoz, Lezcano-Barbero y Colomer-Feliu (2015) en su estudio sobre la revisión de la mentoría en 35 universidades españolas, lo definen como orientación y acompañamiento continuo. Fish (2017) subraya que aumenta la capacidad personal y profesional en el proceso de interacción entre iguales.

La mentoría ha estado ligada a momentos de transición y cambio en la vida de las personas. Las situaciones de transición por las que un individuo va a encontrarse son muy variadas desde personales, educativas, laborales, etc. Si nos centramos en la educación, la transición a la universidad es una de las que más atención se ha venido prestando. Según Moreno (2003) en la educación universitaria existen tres transiciones clave que pueden determinar los espacios temporales en los que es necesario una mayor incidencia de intervención orientadora: a) primera transición: la entrada en la universidad, b) segunda transición: durante la estancia del estudiante en la universidad, y c) tercera transición: al finalizar los estudios. Respecto a la primera transición, las dificultades que el universitario novel presenta están relacionadas con la escasa información sobre sí mismo, sobre el contexto donde ha de desenvolverse, sobre la metodología de estudio y sobre las exigencias de la titulación de grado y/o postgrado en la que se ha matriculado. Supone un tipo de orientación preventiva, de tutoría individualizada para facilitar la toma de decisiones y la adaptación a su nueva situación.

Las universidades deben brindar a los nuevos estudiantes experiencias de aprendizaje que ayuden a cumplir sus expectativas, despertarles el interés y el ánimo para alcanzar los objetivos, que le capaciten para solventar las dificultades que puedan encontrar en el camino para obtener el éxito (Johnston, 2013).

Con el inicio del Espacio Europeo de Educación Superior (EEES), las universidades se comprometieron a diseñar y organizar un plan de acogida y orientación que permitiera a los estudiantes nuevos transitar a esta etapa y minimizar situaciones de abandono generadas por el fracaso académico. Siguiendo a Bisquerra y Álvarez (1998), el aprendizaje centrado en el estudiante requiere de estructuras eficaces de apoyo y orientación.

En el caso de la Universidad Nacional de Educación a Distancia (en adelante, UNED), se apostó por un Plan de Acogida mixto: virtual y presencial, de acuerdo con su peculiaridad de aprendizaje semipresencial o blearning. Sus principios se apoyan en las teorías psicopedagógicas que otorgan la máxima importancia a la autonomía y autorregulación del estudiante en su aprendizaje (Sánchez-Elvira et al., 2006; Zimmerman, 1989). El estudiante como protagonista coresponsable de su propio proceso de aprendizaje, siendo la autonomía y la autorregulación dos características clave para el éxito académico y personal en este paradigma centrado en el estudiante, en el que el docente desarrolla un rol más dirigido a la orientación y la tutoría.

Los planes de acogida emergen como medida de orientación, apoyo y ayuda, principalmente. La UNED, consciente de esta necesidad, y de acuerdo con su planteamiento metodológico, favorece el diseño de programas y cursos estratégicos para la guía y acogida de los nuevos estudiantes (Martín-Cuadrado, 2016). El modelo de Educación a Distancia (en adelante, EaD) de la UNED y el apoyo de la red de Centros Asociados repartidos por toda la península española suponen la oportunidad para realizar una propuesta de bMentoría con los estudiantes de nuevo ingreso, y como actividad programada en el Plan de Acogida de la universidad. Para construir el marco de referencia de esta propuesta, se tomó como punto de partida los resultados de investigaciones realizadas en la UNED. 
Desde el Centro de Orientación y Empleo (en adelante, COIE) se han realizado estudios piloto para la implantación de un programa de Mentoría Universitaria (Sánchez, Manzano, Suárez, Rísquez y Oliveros, 2009) en los que se destaca la necesidad de orientación que tienen los estudiantes.

El proyecto de Orientación y Acción Tutorial constituía un modelo de tutoría y mentoría basado en la intervención educativa y orientadora que se ocupaba de los aspectos académicos de los estudiantes, de la mejora de su rendimiento, de la ampliación de sus expectativas y de la mejora profesional (Manzano-Soto, Martín-Cuadrado, García, Rísquez y Suárez, 2012). Orientar y asegurar un acompañamiento y entrenamiento adecuado es uno de los principales objetivos (Manzano-Soto y Roldán-Morales, 2015).

El proyecto se llevó a cabo en cinco centros asociados de la UNED, con una muestra incidental de 258 mentorizados, 35 compañeros mentores y 18 consejeros. La coordinación del proyecto fue del COIE central (Sánchez, Manzano-Soto, Rísquez y Suárez, 2011).

Durante el curso académico 2011-2012 y 2012-2013, por parte del Vicerrectorado de Internacionales (UNED) surgió una propuesta para formar a e-mentores que se ocuparan del acompañamiento de estudiantes extranjeros de los programas de Cooperación y Voluntariado. Actualmente, se ha formado a una decena de e-mentores que iniciaron su cometido en la Comunidad Virtual de Acogida de Estudiantes de movilidad durante el curso académico 20142015, hecho que refleja la apuesta por esta estrategia (Vargas, González-Brignardello, MartínCuadrado y Valero, 2014).

Los estudios sobre ementoría aportan información útil para la construcción del Programa de bMentoría. Rísquez (2006), presenta la e-mentoría como un complemento y refuerzo de otras formas de apoyo social, emocional, e intelectual, dinámico que implica a todos los participantes en el proceso de auto-aprendizaje, acción y reflexión. Una de las implicaciones de diseño que se desprenden de las conclusiones a las que llega Rísquez (2010) en cuanto al uso de la comunicación para facilitar relaciones de mentoría entre pares, apunta a la adopción de modelos mixtos que combinen el contacto presencial y virtual, cuando esto sea posible.

Ligado a estas investigaciones, interesa destacar el enfoque del aprendizaje basado en el conectivismo (Siemens, 2004) y su relación con el programa de bMentoría. Esta forma de aprender se centra en las posibilidades que ofrece la sociedad actual y el amplio desarrollo de las redes sociales en los grupos de estudiantes (García-Fuentes y Manzano-Soto, 2015). En este sentido nos parece interesante aprovechar estas posibilidades para los estudiantes nuevos, en una universidad como la UNED, que destaca por su desarrollo en los sistemas digitales e Internet.

Moncada (2014) afirma que el factor de integración académica es determinante en el abandono o permanencia de los estudiantes nuevos en los modelos de enseñanza a distancia. El punto de partida no es sólo los encuentros presenciales puntuales, se trata de proveer un ambiente de comunicación flexible, independiente de barreras de espacios y tiempo. Se puede establecer una comunicación asincrónica y sincrónica a través de las TIC (Cabero, Cebrián, Duarte, Gisbert, Martínez y Salinas, 2000). En el enfoque blearning se combina la enseñanza presencial con la tecnología no presencial, determinar qué parte puede ser de autoaprendizaje y de tutoría, qué parte sincrónica y que tiempo asincrónico (Arias, 2011), para rentabilizar el estudio de los estudiantes nuevos y así utilizar lo mejor de la parte presencial y de la virtual (Rísquez, 2010). Los entornos de aprendizaje, las relaciones personales y experiencias han experimentado toda una revolución a través de las redes sociales, los límites de la mentoría no se circunscriben a los ámbitos formales, sino también a los no formales e informales. Incrementar las posibilidades educativas de los futuros titulados aprovechando estas nuevas coordenadas espacio-temporales es una posibilidad que no debe caer en el olvido (Salinas, 2012). 
Nuestra apuesta es el modelo de bMentoría en el contexto del Centro Asociado en la UNED, de manera presencial y online apoyado en las redes sociales. El propósito es proporcionarles habilidades para conseguir el éxito en sus estudios y mejoras en el rendimiento académico (Martín-Cuadrado, 2016).

\section{Problema y objetivos de investigación}

El problema de investigación que nos planteamos está relacionado con el rendimiento académico y las elevadas tasas de abandono que se producen durante los primeros cursos académicos universitarios, siendo conscientes de la necesidad de valorar todos los aspectos que llevan a esta situación. Pensamos que existe una relación significativa entre la puesta en marcha de un programa de bMentoría con estudiantes nóveles universitarios durante los dos primeros cursos académicos y el satisfactorio rendimiento académico.

El objetivo general es el diseño y validación de un modelo de bMentoría Universitaria en el Centro Asociado de la UNED en Sevilla, como medida de orientación y seguimiento de los estudiantes de nuevo ingreso, que les permita terminar sus estudios con adecuadas tasas de rendimiento académico-

\section{Respecto al programa}

- Recopilar información documental para elaborar el marco teórico conceptual sobre la construcción de un programa de bMentoría universitaria en Centros Asociados de la UNED.

- Describir el funcionamiento del programa de bMentoría en base a la modalidad mixta, combinando la presencialidad con la virtualidad.

- Descubrir las debilidades y fortalezas del programa de bMentoría.

- Evaluar el contexto, el proceso y el producto del programa, recogiendo las propuestas, experiencias y aportaciones de los participantes.

- Identificar perfiles de estudiantes en función de las dificultades encontradas al inicio de los estudios

- Validar la metodología de la bMentoría adaptado al Centro Asociado de Sevilla en lo concerniente a todas sus fases.

En este artículo, presentamos los resultados del programa de bMentoría durante el primer año de implementación, teniendo en cuenta algunas dimensiones y variables influyentes en la comunicación entre los bmentores y bmentorizados, así como las dificultades que encuentran los estudiantes nuevos cuando se incorporan a la UNED.

\section{Descripción del Programa de bMentoría}

El Programa de Orientación Tutorial y bMentoría, se sustenta en el modelo de tutoría entre iguales, en el que los estudiantes veteranos tutorizan a los más noveles para el desarrollo de habilidades y competencias necesarias para lograr el buen rendimiento académico. Este Programa es una adaptación del proyecto piloto de mentoría que se realizó en la UNED durante los cursos académicos 2007-2008 y 2008-2009 (Sánchez, et al., 2009). La experiencia alcanzada por las diversas investigaciones al respecto, reflejan que es muy recomendable su implantación (Allcueva, 2013; Benavent y Fossati, 1990; Casado y Ruiz, 2009; Castellano y Delgado, 1996; Dewart et al., 2004; Dubon, Climent, García y Romero, 2001; Miller, 2002; Dubon, Climent Pakhrou, Gil, Abad, y Martínez, 2011; Rísquez y Moore, 2005; Sánchez, et al.; 2009; Valverde, Ruiz, García y Romero 2003; Vega, 2000; Yusta, Cepero, Prieto, Abadía y Bueno, 2015) 
El desarrollo de las relaciones interpersonales que se generan entre los implicados en el proceso de bMentoría, se produce en base a unos objetivos concretos y con una metodología definida que sirve a los propósitos que, previamente, se acuerdan. En las relaciones interpersonales informales, el apoyo y la interacción surgen de manera espontánea; en la bMentoría, el objetivo está definido previamente.

En el Programa de bMentoría destacan dos figuras principales: los estudiantes bmentorizados (reciben apoyo para su integración académica) y los estudiantes bmentores (ofrecen ayuda y soporte). Para estos últimos, supone, también un instrumento para adquirir y desarrollar competencias personales y profesionales muy valoradas en el mercado de trabajo (capacidad de comunicación, iniciativa personal, liderazgo, etc.), por lo que podríamos afirmar que para los bmentores el programa se convierte en una herramienta de mejora de su inserción y desarrollo profesional.

El programa facilitaría el proceso de transición y adaptación de los estudiantes de nuevo ingreso; además, provee la configuración de un itinerario curricular atendiendo a las necesidades y especificidades de los estudiantes. El modelo bMentoría que se defiende en esta investigación es un Programa de Orientación Tutorial y bMentoría que se desarrolla como un proceso de ayuda, orientación, acompañamiento e interrelación personal, en el que un estudiante con competencias interpersonales y experiencia académica es capaz de favorecer el aprendizaje e inclusión en la universidad de estudiantes nuevos, alternando el contacto entre las redes sociales y el contexto presencial. La experiencia piloto se inició durante el curso 2014-2015.

\section{El programa de bMentoría en el Centro Asociado de la UNED en Sevilla}

El centro asociado de la UNED en Sevilla viene realizando su labor desde 1985. La ciudad cuenta con, aproximadamente, 1.938 .765 habitantes y está rodeada de otras poblaciones más pequeñas, 105 municipios. El 35,5\% de los habitantes (INE, 2016) tienen edades comprendidas entre los 20-44 años. La matrícula en el centro asociado ronda los 6000 estudiantes.

\section{Objetivos del Programa}

El objetivo de esta propuesta como medida de orientación y seguimiento de los estudiantes de nuevo ingreso surge por el interés en mejorar el rendimiento académico y reducir las cifras de abandono durante la primera matrícula (Callejo, 2001; García-Aretio, 1987; Luque-Pulgar, GarcíaCerdeño y Santiago, 2013; Manzano-Soto et al., 2012; Santiago de, 2011). De acuerdo con algunas de las investigaciones realizadas en la UNED (Sánchez-Elvira et al., 2006) sobre estrategias de aprendizaje, puntualizan que el éxito de estudiantes nuevos en la UNED se debe a organización de la información, persistencia, procesamiento profundo, pautas fijas de estudio. Con respecto al fracaso, tiene que ver con la ansiedad, dificultad para organizar el tiempo, evitación de dificultades, procesamiento superficial, etc.

\section{Destinatarios del Programa}

Los destinatarios de este programa son los estudiantes del Centro Asociado de la UNED de Sevilla:

- bMentores

- bMentorizados

- Egresados

- Profesionales en activo

El bmentor es un estudiante que se caracteriza por su disponibilidad continua para atender sincrónica y asincrónicamente a los bmentorizados, tanto en el plano presencial como virtual a 
través de los espacios habilitados para tal fin; y, poseer habilidades de escucha activa, empatía, cordialidad y veracidad. Es capaz de crear un clima de confianza, demostrando interés, aceptación y valor por los estudiantes que atiende. Por otra parte, deben participar activamente en la actividad educativa del centro asociado, obteniendo el reconocimiento por el resto de la comunidad universitaria (Corral-Carrillo y Martín-Cuadrado, 2017).

El bmentorizado es un estudiante de primer año, que percibe la necesidad de apoyo y orientación académica. El Programa de Orientación Tutorial y bMentoría supone una respuesta a sus necesidades.

El estudiante egresado ofrece su disponibilidad a colaborar y ayudar a los estudiantes nuevos y a los bmentores. Es conocedor del Programa y mantiene vínculos con el Centro Asociado participando en actividades diversas.

Los profesionales en activo realizan una labor de motivación hacia los estudiantes nuevos, ofreciendo la oportunidad de visitar los espacios de trabajo y participando en seminarios prácticos sobre el desarrollo de la identidad profesional, el emprendimiento y la empleabilidad.

\section{Diseño del Programa}

Por otra parte, el programa está diseñado desde un enfoque blearning, siguiendo el modelo de enseñanza aprendizaje de la UNED, rentabilizando los recursos de apoyo y superando las dificultades que pudieran tener algunos estudiantes para coincidir en el centro asociado y además, aprovechar la fluidez de lo virtual desde la potencialidad de las redes sociales (Corral-Carrillo y Martín-Cuadrado, 2015).

\section{Estrategias metodológicas}

Este programa supone una acción más en el Plan de Acogida que se lleva a cabo en el Centro Asociado de la UNED en Sevilla. La estructura del Programa de Orientación Tutorial y bMentoría tiene como eje central y potenciador al COIE, como servicio que ofrece soporte y ayuda a los estudiantes universitarios su adaptación e integración académica y para facilitar la inserción laboral y desarrollo profesional de los graduados y posgraduados. Una persona ejerce la coordinación del programa, llevando a cabo todas las acciones que se precisan. Los bmentores son los agentes protagonistas de esta iniciativa, dando cobertura a los estudiantes nuevos que solicitan ser bmentorizados.

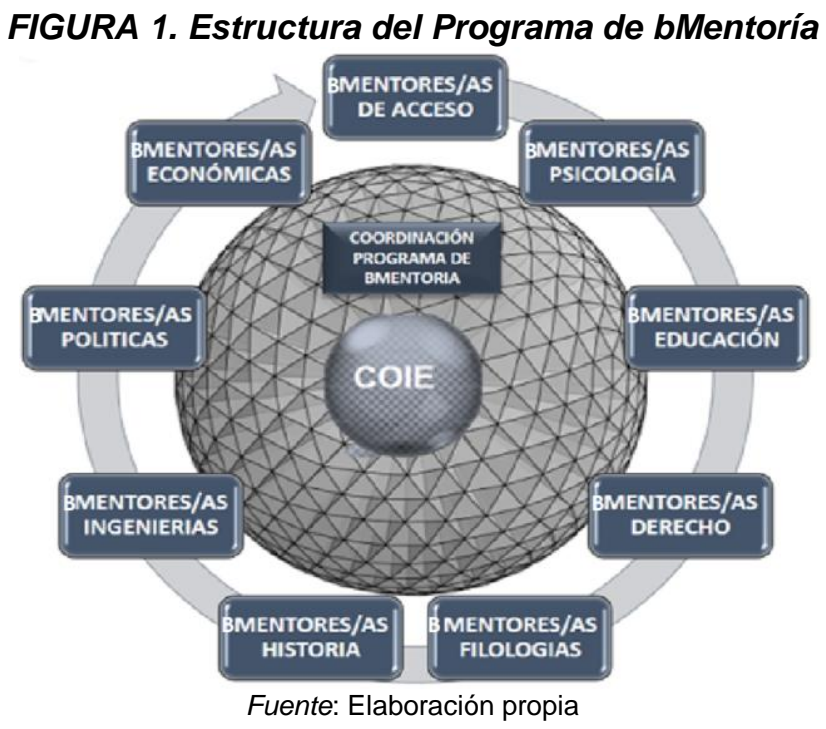


Este programa combina diferentes métodos y estrategias, se aplica en diferentes momentos, al inicio de cada semestre, y de acuerdo con las diferentes situaciones: desde la presencialidad, (en el Centro Asociado en Sevilla), a la virtualidad en contextos dispares (plataforma UNED y redes sociales). La metodología se apoya en el aprendizaje y tutorización entre pares y el desarrollo del aprendizaje autónomo del estudiante (Álvarez y González, 2005). Se persigue el aprendizaje colaborativo, el aprender de/con otros, promoviendo la interdependencia al mismo tiempo que la autonomía y autorregulación (Jarauta, 2014). A través del aprendizaje cooperativo-colaborativo se logra el desarrollo de competencias necesarias para el mejor aprovechamiento, no sólo para los estudiantes de nuevo ingreso, sino también para los estudiantes que ejercen de bmentores.

\section{Método}

La investigación que presentamos se plantea como un estudio descriptivo y explicativo que sirve para mostrar la valoración de la plataforma aLF y la utilización de los medios de comunicación en la relación de bMentoría, así como las dificultades encontradas como estudiantes de la UNED, durante su primer año. Dado que el estudio es de corte cuantitativo, se aplicaron dos cuestionarios: uno, a los estudiantes bmentores y otro, a los estudiantes bmentorizados. Para poder describir y explicar el objeto de estudio se seleccionaron, midieron y analizaron estadísticamente algunas de las dimensiones planteadas en los cuestionarios.

\section{Muestra}

La población se corresponde con los estudiantes del Centro Asociado de la UNED en Sevilla, en el curso académico 2014/2015. La muestra de estudiantes bmentores se ha obtenido en base a un muestreo no probabilístico intencional o de conveniencia, siempre que se cumpliera con el perfil del mentor, descrito en investigaciones anteriores (Manzano-Soto et al., 2012). En algunos de los grados académicos no se obtuvieron estudiantes bmentores para atender la demanda, y se seleccionaron bmentores de grados afines.

En la tabla 1 se puede apreciar el número de estudiantes bmentores que se inscribieron y los que, finalmente, concluyeron el programa.

TABLA 1. Muestra de estudiantes bmentores participantes en el Programa

\begin{tabular}{lccc}
\hline & Hombres & Mujeres & Total \\
\cline { 2 - 4 } Inscripción Inicial & 28 & 31 & 59 \\
Culminan el programa & 13 & 20 & 33 \\
\hline \multicolumn{3}{c}{ Fuente: Elaboración propia }
\end{tabular}

Los estudiantes bmentorizados son estudiantes de nuevo ingreso y manifiestan interés por recibir un programa de acompañamiento durante el curso académico, de forma voluntaria. El muestreo utilizado para su selección es probabilístico ya que todos los sujetos de la población tenían las mismas posibilidades de ser parte de la muestra (Tabla 2) 
TABLA 2. Muestra de estudiantes inscritos en el Programa (bmentores y bmentorizados)

\begin{tabular}{lccc}
\hline \multicolumn{1}{c}{ Plan de Estudios } & Matricula & bmentores & bmentorizados \\
\hline Grado en Economía & 130 & 1 & 3 \\
Grado en Administración y Dirección de Empresa & 461 & & 14 \\
Grado en Geografía e Historia & 253 & 1 & 4 \\
Grado en Turismo & 95 & & 6 \\
Grado en Ingeniería Informática & 137 & 1 & 3 \\
Grado en Trabajo Social & 280 & & 4 \\
Grado en Pedagogía & 126 & 10 & 16 \\
Grado en Ciencia Política y de la Administración & 144 & 1 & 5 \\
Grado en derecho & 998 & 3 & 29 \\
Grado en Lengua y Literatura Española & 94 & & 2 \\
Grado en Estudios ingleses: lengua, literatura y cultura & 141 & & 7 \\
Grado en Historia del Arte & 212 & & 11 \\
Grado en Educación Social & 222 & 6 & 3 \\
Grado en Sociología & 78 & 1 & 31 \\
Grado en Ingeniería en Tecnologías de la Información & 53 & & 5 \\
Grado en Psicología & 1398 & 9 & \\
Grado en Ciencias Jurídicas de la Administración Pública. & 1078 & & \\
Diplomatura en Trabajo Social & 8 & & \\
Licenciatura en Psicología & 25 & & \\
Licenciatura en Pedagogía & 25 & & \\
Diplomatura en Turismo & 4 & & \\
Licenciatura en Derecho & 93 & & \\
CUID & 375 & & \\
Acceso a mayores de 25 años & 477 & & \\
TOTAL & 6123 & 33 & \\
\hline
\end{tabular}

Fuente: Elaboración propia

\section{Dimensiones, categorías y variables a evaluar}

A continuación, se describen las dimensiones, categorías y variables:

Para bmentores

- Espacio de comunicación

- Valoración de la plataforma UNED (aLF)

- Valoración de la utilización de los medios de comunicación (a través del foro virtual, en el centro asociado, por correo electrónico, por teléfono, espacio informal cafetería, WhatsApp, Facebook, Twitter, Linkedln y Skype).

Para bmentorizados 
- Espacio de comunicación

- La relación y comunicación con los bmentores (a través del foro virtual, en el centro asociado, por correo electrónico, por teléfono, espacio informal cafetería, WhatsApp, Facebook, Twitter, Linkedln y Skype).

- Las dificultades encontradas como estudiantes de la UNED (dificultades para organizar y planificar tu tiempo de estudio, dificultades para utilizar estrategias/técnicas de estudio, dificultades para simultanear tu actividad de estudio con otras ocupaciones, aspectos concretos de asignaturas, desconocer los planes de estudio, inseguridad, baja autoconfianza, alta motivación, aspectos burocráticos, falta de información sobre aspectos organizativos de la Universidad y Facultad, desconocimiento y falta de uso de los recursos a tu alcance, desconocer las salidas profesionales de tu carrera, no conocer a tus compañeros o no tener confianza con ellos)

\section{Instrumentos}

Para la recogida de datos se adaptaron dos cuestionarios elaborados por Sánchez et al. (2009). En los cuestionarios resultantes se mantuvieron las principales dimensiones recogidas en los cuestionarios originales, aunque algunos ítems se eliminaron, otros se incluyeron o se modificaron en función de los objetivos planteados.

El cuestionario del programa de bMentoría para compañeros bmentores estaba compuesto por 24 ítems, de corte cuantitativo (escala tipo Likert con cuatro y cinco posibles respuestas), que certifican la consistencia interna a partir del análisis de fiabilidad en cada dimensión y globalmente (a de Cronbach=.87). En la tabla 3 se aprecian las dimensiones, así como el número de categorías y variables. En esta ocasión, el estudio se ha centrado en la dimensión, el espacio de comunicación.

TABLA 3. Dimensiones, categorías y variables. Estudiantes bmentores

\begin{tabular}{lcc}
\hline Dimensiones & Categorías & Variables \\
\hline Datos situacionales & 1 & 2 \\
Relación de Mentoría & 3 & 12 \\
Opinión sobre el programa de bMentoría & 8 & 8 \\
Formación Recibida & 2 & 2 \\
Espacio de comunicación & 4 & $\mathbf{1 8}$ \\
$\begin{array}{l}\text { Beneficios percibidos con la participación en el } \\
\text { programa de bMentoría }\end{array}$ & 23 \\
\hline \multicolumn{2}{c}{ Fuente: Elaboración propia }
\end{tabular}

El cuestionario del programa de bMentoría para compañeros bmentorizados estaba compuesto por 7 ítems de corte cuantitativo (escala tipo Likert con cuatro y cinco posibles respuestas), que certifican la consistencia interna a partir del análisis de fiabilidad en cada dimensión y globalmente ( $\alpha$ de Cronbach=.87). En la tabla 4 se aprecian las dimensiones, así como el número de categorías y variables. En esta ocasión el estudio se ha centrado en dos dimensiones: el espacio de comunicación y las dificultades encontradas como estudiantes nuevos. 
TABLA 4. Dimensiones, categorías y variables. Estudiantes bmentorizados

\begin{tabular}{lcc}
\hline Dimensiones & Categorias & Variables \\
\hline Datos situacionales & 1 & 2 \\
Espacio de comunicación & 2 & 8 \\
Consultas y preguntas & 1 & 11 \\
Dificultades encontradas & 1 & 9 \\
Opinión sobre el programa de bMentoría & 1 & 2 \\
\hline \multicolumn{2}{c}{ Fuente: Elaboración propia }
\end{tabular}

\section{Procedimiento}

La puesta en marcha de la experiencia se llevó a cabo durante el curso académico 2014/2015. Estaba respaldada por la coordinación del COIE y por la dirección del centro asociado de la UNED en Sevilla.

Una vez adaptados los cuestionarios, validados y realizado el análisis de su fiabilidad, se utiliza la herramienta formularios de Google Drive para la maquetación, envío vía internet y recuperación posterior de datos.

La aplicación de los cuestionarios se llevó a cabo a través de dos vías:

- Envío del enlace del cuestionario de Google Drive a los bmentores a través del correo electrónico de estudiante de la UNED.

- Envío del enlace del cuestionario de Google Drive a los bmentores a través de WhatsApp

Los estudiantes bmentores se encargaron de enviar el cuestionario a sus respectivos bmentorizados a través de los grupos de WhatsApp. Aquellos estudiantes bmentorizados que manifestaban dificultad en realizar el cuestionario en formato digital, lo realizaron con los bmentores en formato papel.

\section{Resultados}

Se ha utilizado un método de análisis de los estadísticos descriptivos y de frecuencia para hallar las características de las variables estudiadas de corte cuantitativo, con el paquete estadístico SPSS 22.0. El nivel de respuestas de los participantes en el programa es del 84,8\% para bmentores y del $73 \%$ para bmentorizados.

\section{bmentores}

La variable relacionada con los espacios virtuales utilizados en la relación de bMentoría es uno de los aportes más representativo en esta investigación.

Se construyó una comunidad virtual mediada por las tecnologías, para que los integrantes del programa de bMentoría pudieran contactar, con objetivos comunes, lo que incrementaba la 
interacción por la propia necesidad de intercambio. El diseño de esta comunidad de aprendizaje virtual consistió en un espacio en abierto en la plataforma de la UNED (aLF); es decir, los participantes pueden ser usuarios UNED o no, principalmente por las características de sus integrantes: egresados y en algunos casos, profesionales en activo (Corral-Carrillo y MartínCuadrado, 2015).

La valoración de la plataforma se realizó a través de una serie de preguntas que exigían una respuesta con una escala de tipo Likert de cuatro puntos (muy desfavorable, desfavorable, favorable y muy favorable). Se encontró que las respuestas positivas suman el $75 \%$ del total (ver figura 2).

FIGURA 2. Valoración de la Plataforma aLF

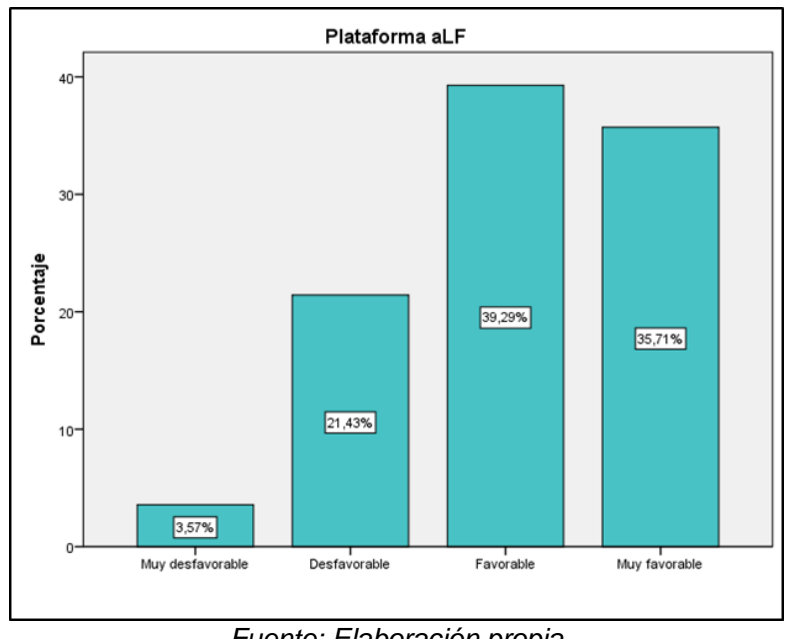

Fuente: Elaboración propia

En la tabla 5, se analizan las preferencias de comunicación del bmentor con los estudiantes asignados. Se observa que el WhatsApp y el correo electrónico son los medios preferidos, con un porcentaje de respuesta del $56 \%$ y el $40 \%$, respectivamente. El foro virtual de la comunidad es otro canal utilizado por el $32 \%$ de los casos.

TABLA 5. Preferencia de comunicación de los estudiantes bmentores

\begin{tabular}{lc}
\hline \multicolumn{1}{c}{ Preferencia de comunicación } & Porcentaje de casos \\
\hline A través del foro virtual & $32,0 \%$ \\
En el centro asociado & $16,0 \%$ \\
Por correo electrónico & $40,0 \%$ \\
Por teléfono & $20,0 \%$ \\
Espacio informal cafetería, etc. & $12,0 \%$ \\
Redes sociales: WhatsApp & $56,0 \%$ \\
Redes sociales: Facebook & $8,0 \%$ \\
Redes sociales: Twitter & $8,0 \%$ \\
Redes sociales: Linkedln & $8,0 \%$ \\
Redes sociales: Skype & $8,0 \%$ \\
\hline \multicolumn{2}{c}{ Fuente: Elaboración propia } \\
\hline
\end{tabular}

El proceso de relación entre el bmentor y el bmentorizado no se produce exclusivamente en el contexto formal, sea virtual o presencial. Existen espacios no formales e informales que favorecen 
experiencias enriquecedoras en las que los estudiantes reflexionan juntos y con otras personas, compartiendo y construyendo conocimientos (Martín-Cuadrado, 2011).

En la tabla 4 se valora la utilización real de los medios de comunicación con una escala tipo Likert de cinco puntos (nada, poco, regular, casi siempre y siempre).

Los estudiantes bmentores han respondido a sus estudiantes bmentorizados mayoritariamente a través del WhatsApp, con un 92,9\%, aunque manifiestan preferir la combinación con otros medios. Se observa que los espacios presenciales en el centro asociado y otros espacios más informales, como la cafetería, pasillos, etc. gozan de una valoración bastante positiva. La temática a resolver influye en este tipo de valoraciones, tal como indican los bmentorizados en las sesiones grupales.

TABLA 6. Valoración de la utilización de los medios de comunicación

\begin{tabular}{lccccc}
\hline & $\begin{array}{c}\text { Nada } \\
\%\end{array}$ & $\begin{array}{c}\text { Poco } \\
\%\end{array}$ & $\begin{array}{c}\text { Regular } \\
\%\end{array}$ & $\begin{array}{c}\text { Casi Siempre } \\
\%\end{array}$ & $\begin{array}{c}\text { Siempre } \\
\%\end{array}$ \\
\hline A Través del Foro virtual & 25 & 28,6 & 32,1 & 14,3 & 0 \\
En el Centro Asociado & 3,6 & 10,7 & 21,4 & 35,7 & 14,3 \\
Por Correo Electrónico & 0 & 3,6 & 10,7 & 32,1 & 53,6 \\
Por teléfono & 3,6 & 10,7 & 3,6 & 28,6 & 46,4 \\
Espacio Informal Cafetería, etc. & 3,6 & 7,1 & 32,1 & 25 & 32,1 \\
Redes Sociales: WhatsApp & 7,1 & 0 & 0 & 0 & 92,9 \\
Redes Sociales: Facebook & 17,9 & 14,3 & 14,3 & 46,4 & 7,1 \\
Redes Sociales: Twitter & 75 & 10,7 & 7,1 & 7,1 & 0 \\
Redes Sociales: Linkedln & 96,4 & 3,6 & 0 & 0 & 0 \\
Redes Sociales: Skype & 100 & 0 & 0 & 0 & 0 \\
Otro & 85,7 & 7,1 & 0 & 0 & 0 \\
\hline
\end{tabular}

\section{bmentorizados}

En primer lugar, se ha estudiado la frecuencia con la que los estudiantes bmentorizados se han comunicado con sus respectivos bmentores y a través de qué medios lo han hecho. El 36,4\% manifiesta haberse comunicado una vez al mes, mientras que el $35,6 \%$ se ha comunicado una vez a la semana; el 11,9\% afirma haberse comunicado con su bmentor más de una vez a la semana. Con respecto al medio de comunicación, tenían varias opciones (foro, correo electrónico, cara a cara, teléfono y WhatsApp). Se podía elegir entre cuatro posibles respuestas $10 \%, 20 \%, 30 \%$ y $40 \%$. De manera general, se ha realizado un análisis de diferencia de medias para discernir el medio de comunicación más utilizado. La tabla 7 muestra dicho análisis, y se puede comprobar que el WhatsApp es el medio mayormente utilizado seguido por el correo electrónico.

TABLA 7. ¿Con qué frecuencia te has comunicado con tu bmentor/a?

\begin{tabular}{lccccc}
\hline & A través del foro virtual & Por correo electrónico & $\begin{array}{c}\text { Cara a } \\
\text { cara }\end{array}$ & Por teléfono & $\begin{array}{c}\text { Por } \\
\text { WhatsApp }\end{array}$ \\
\hline Media & 10,85 & 31,19 & 21,10 & 21,61 & 37,20 \\
$\begin{array}{l}\text { Desviación } \\
\text { estándar }\end{array}$ & 4,046 & 11,336 & 11,678 & 11,765 & 7,027 \\
\hline \multicolumn{7}{c}{ Fuente: Elaboración propia }
\end{tabular}


Durante el desarrollo del programa, han surgido diversas consultas entre los participantes, que han servido para realizar un análisis y averiguar qué tipo de cuestiones eran las que más dudas planteaban entre los bmentorizados. La consulta menos significativa fue relativa a las relaciones y el trato entre los compañeros; en este sentido, los estudiantes se relacionan sin dificultad. Por otra parte, las dudas más relevantes versan sobre las asignaturas, en general, y sobre los profesores y su metodología de enseñanza (ver figura 3).

\section{FIGURA 3. El tipo de preguntas realizadas al compañero bmentor por el compañero bmentorizado}

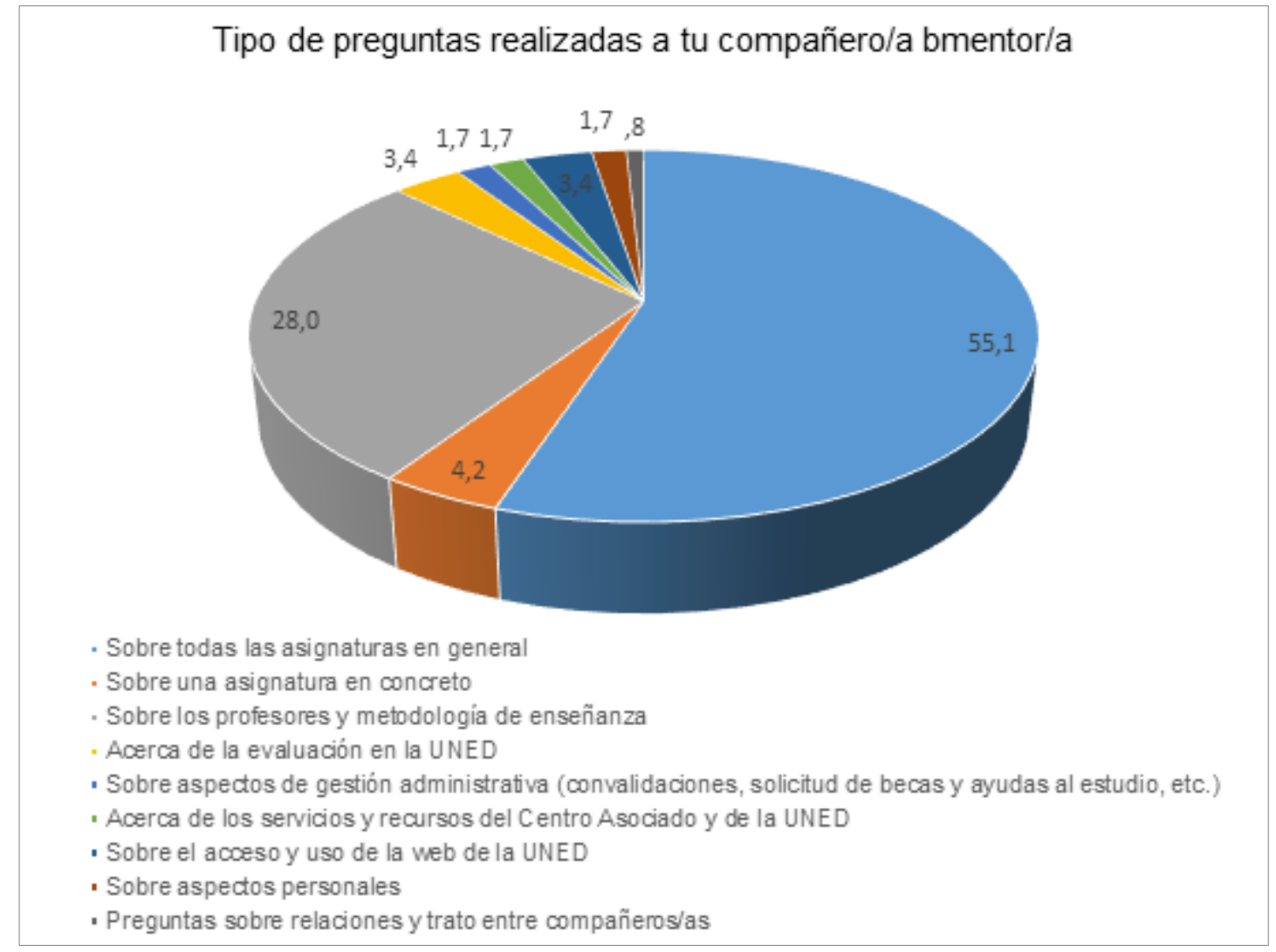

Fuente: Elaboración propia

El asesoramiento y orientación a la hora de afrontar el estudio de las diferentes asignaturas ocupa gran parte del tiempo de los estudiantes bmentores. En muchas ocasiones, los estudiantes nuevos se sienten desbordados por la cantidad de material de los cursos virtuales y no llegan a saber cómo afrontar las asignaturas.

Se estudian las dificultades que pudieron surgir a los bmentorizados, obteniéndose que el $62,7 \%$ tuvo alguna dificultad a lo largo del año académico, el $24.6 \%$ tuvo poca dificultad en los diferentes aspectos estudiados, y el $12.7 \%$ mucha dificultad (ver figura 4). Esto puede ser debido a la implementación del programa de bMentoría, ya que los estudiantes veteranos ayudan y acompañan en las dificultades que los estudiantes nuevos se van encontrando. 
FIGURA 4. Dificultades encontradas como estudiante de la UNED

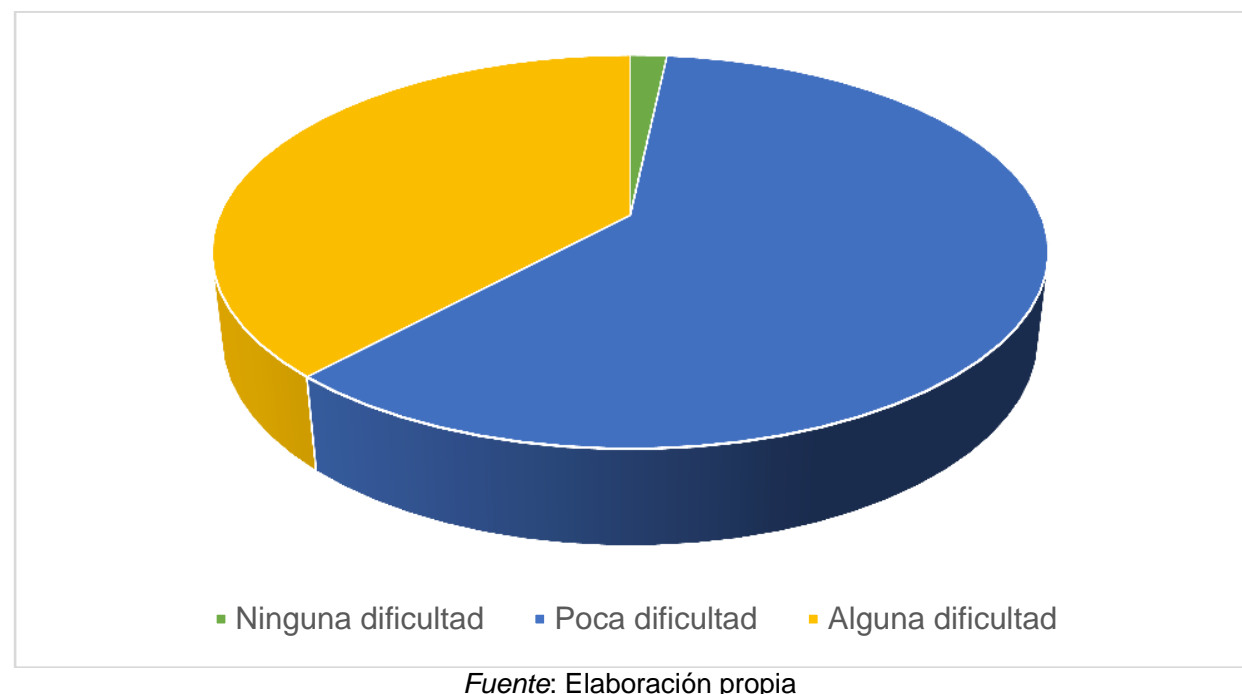

Fuente: Elaboración propia

En la tabla 8 destacan, como tendencia más generalizada, las dificultades que tienen los estudiantes para simultanear los estudios con otras ocupaciones. Otras dificultades importantes, son: por un lado, los estudiantes presentan muchas dudas respecto a aspectos concretos de las asignaturas, y por otro, desconocimiento para utilizar estrategias y técnicas de estudio apropiadas.

TABLA 8. Promedio y desviación típica de las dificultades encontradas como estudiante de la UNED

\begin{tabular}{lcc}
\hline \multicolumn{1}{c}{ Variable } & Media & Desv. típica \\
\hline Dificultades para organizar y planificar tu tiempo de estudio & 3,077 & 0,911 \\
Dificultades para utilizar estrategias/técnicas de estudio & 3,265 & 0,977 \\
Dificultades para simultanear tu actividad de estudio con otras ocupaciones & 3,487 & 0,795 \\
Aspectos concretos de asignatura & 3,376 & 0,926 \\
Desconocer los planes de estudio & 2,940 & 1,132 \\
Inseguridad, baja autoconfianza & 1,530 & 0,664 \\
Falta de motivación & 1,299 & 0,673 \\
Aspectos burocráticos & 1,573 & 0,746 \\
Falta de información sobre aspectos organizativos de la Universidad y Facultad & 2,632 & 0,961 \\
Desconocimiento y falta de uso de los recursos a tu alcance & 2,906 & 1,083 \\
Desconocer las salidas profesionales de tu carrera & 1,350 & 0,746 \\
No conocer a tus compañeros o no tener confianza con ellos & 1,462 & 0,726 \\
Otras & 1,248 & 0,628 \\
\hline
\end{tabular}

Fuente: Elaboración propia

Se realizó un análisis de conglomerados para determinar las dificultades, utilizando una escala tipo Likert de cuatro puntos (1-ninguna dificultad, 2, 3, 4-mucha dificultad).

En los casos de 1 o 2 de la escala se asumió la no dificultad como estudiante de la UNED; mientras que, cuando se obtuvo 3 o 4 puntos, se consideró que existía dificultad.

El análisis de conglomerados permitió clasificar a los bmentorizados en tres grupos (ver figura 5). 
FIGURA 5. El tipo de preguntas realizadas al compañero bmentor por el compañero bmentorizado

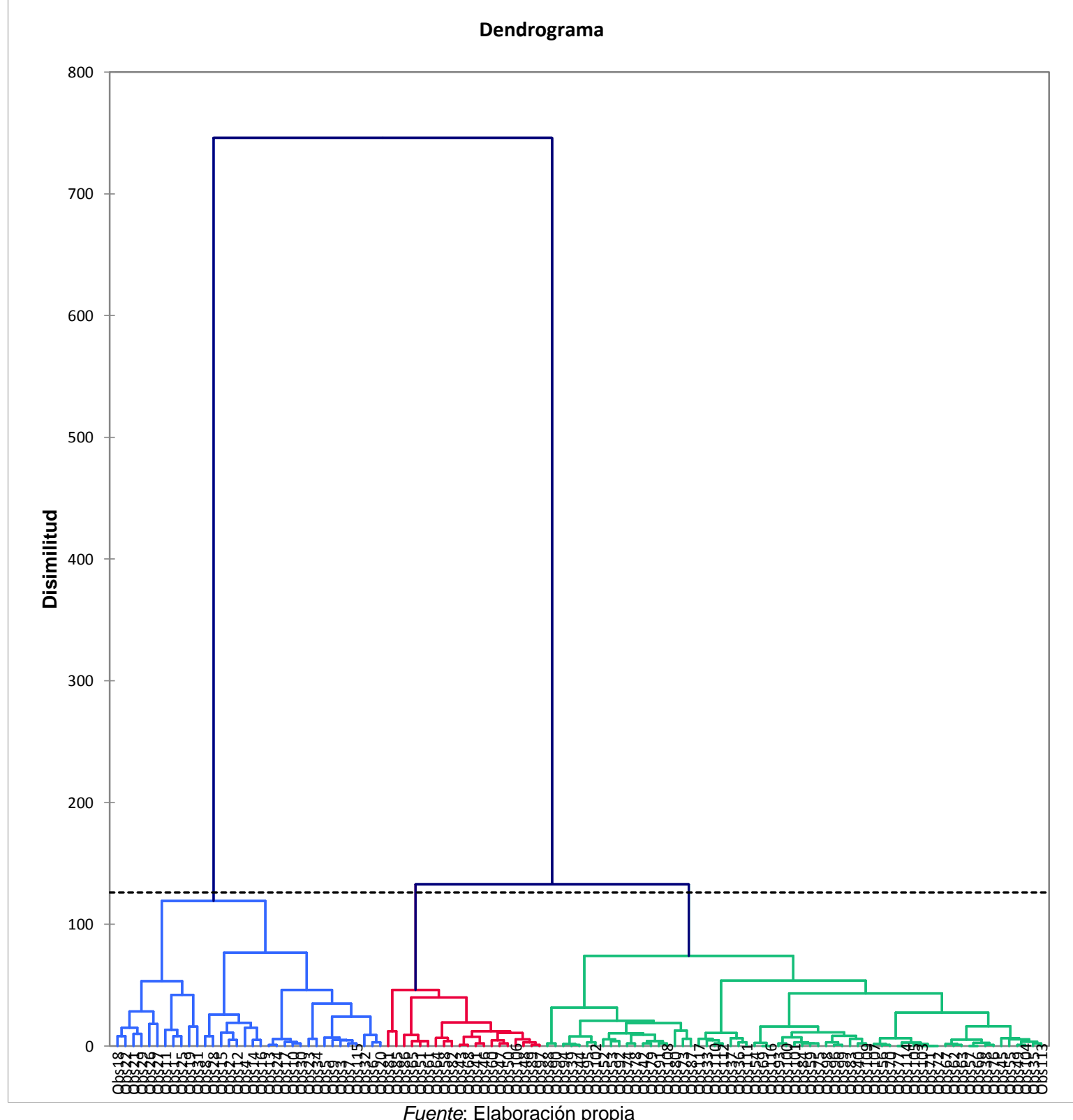

\section{Caracterización del grupo 1}

El grupo que menos dificultades ha encontrado lo componen el $29 \%$ de los estudiantes bmentorizados. Solo, encontraron gran dificultad en simultanear su actividad de estudio con otras ocupaciones. En las sesiones presenciales manifestaban los problemas personales y profesionales que les impedían dedicar más tiempo a los estudios. Dificultades relacionadas con la competencia de gestión y organización del tiempo. 


\section{Caracterización del grupo 2 y 3}

El grupo 2 y 3 se han unido, pues tienen dificultades en las mismas áreas; aunque, el grupo 3 con un punto más en todos los ítems.

Los grupos 2 y 3 encontraron mucha dificultad en los siguientes ítems: el $76,3 \%$ de los estudiantes encuentra dificultades para organizar y planificar el tiempo de estudio, el $78 \%$ para utilizar estrategias/técnicas de estudio, el $88,1 \%$ tiene dificultad en simultanear la actividad de estudio con otras ocupaciones, el 83,1\% encuentra dificultades con los aspectos concretos de asignaturas, y finalmente, los planes de estudio se desconocen por un 69,5\% de los estudiantes.

El 57,6 \% encuentra dificultad en la falta de información sobre aspectos organizativos de la universidad y facultad, y el 66,9\% tiene dificultades con el desconocimiento y falta de uso de los recursos a su alcance. Las dificultades están relacionadas con causas institucionales, educativas y psicológicas, principalmente (García-Aretio, 1994).

En la figura 6 se aprecian los resultados de la valoración del estudiante bmentorizado a la ayuda prestada por el estudiante bmentor. El $55 \%$ de los estudiantes bmentorizados afirman que el estudiante bmentor le ha ayudado mucho a superar las dificultades y el $33 \%$ indica que le ha ayudado bastante; es decir, un porcentaje del $88 \%$ de estudiantes bmentorizados se sienten satisfechos con su bmentor. En este sentido, aunque el trato entre iguales es simétrico y horizontal, existe cierta diferencia de trato en la relación de bMentoría. Se añade que el estudiante de la UNED que elige de forma voluntaria participar como bmentor está especialmente cualificado y motivado para ayudar a los compañeros de primeros cursos.

\section{FIGURA 6. Valoración de los estudiantes nuevos de la ayuda recibida}

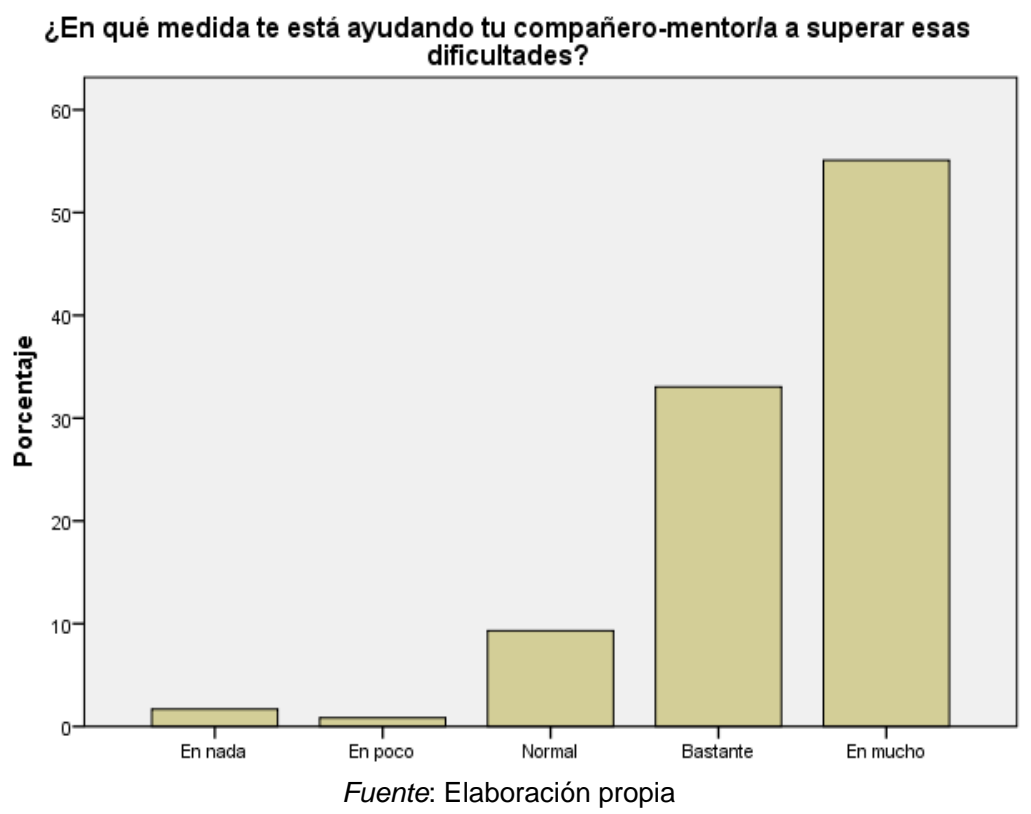




\section{Conclusiones}

En este trabajo se han presentado algunos de los avances en el diseño, desarrollo y evaluación de la puesta en marcha del Programa Orientación Tutorial y bMentoría durante el curso académico 2014-2015 en el Centro Asociado de la UNED en Sevilla.

Las conclusiones que se pueden extraer de este estudio se organizan en torno a dos aspectos: a) dificultades encontradas por los estudiantes bmentorizados durante el primer año en la universidad, y b) medios de comunicación utilizados durante la relación de bMentoría

Las dificultades encontradas por los estudiantes nuevos son las que han tenido que ver con el desconocimiento del plan de estudio, la información y el estudio de las asignaturas, la forma de comunicarse con el equipo docente, la relación existente entre el profesor tutor y el docente, etc. Los bmentores transmiten a sus bmentorizados gran confianza, ya que han cursado esas mismas asignaturas, y facilitan información y asesoramiento personalizado para afrontar el primer momento. Las dificultades que más destacan los estudiantes son las que tienen que ver, principalmente, con simultanear la actividad de estudio y el resto de las ocupaciones; en segundo lugar, dificultades relacionadas con aspectos concretos de asignatura. Un diagnóstico preciso de los aspectos que generan mayor dificultad en las asignaturas de los diferentes grados y un análisis de las estructuras de los cursos virtuales, permitirán diseñar estrategias específicas para disminuir estas dificultades. En tercer lugar, desconocimiento de estrategias y técnicas de estudio adecuadas; y, en último lugar, dificultades para organizar y planificar el tiempo de estudio. Las necesidades detectadas son similares a otras investigaciones realizadas para estudiar el abandono en los estudiantes de la UNED (Callejo, 2001; García-Aretio, 1994; Sánchez-Elvira et al., 2006; Sánchez et al., 2009). Se debe seguir avanzando en el diseño de un plan de acogida y orientación integral que incluya medidas coordinadas desde la UNED y en unión con los centros asociados. Un plan con diferentes niveles de concreción, niveles macro y niveles micro. El objetivo es que el estudiante nuevo consiga realizar los estudios que eligió de acuerdo con su plan personal, académico y profesional.

En relación con la valoración de los espacios de comunicación, la mayoría se comunica una vez a la semana y una vez al mes; el medio más utilizado es el WhatsApp, junto con el correo electrónico a la vez que presencialmente. El WhatsApp al ser un medio de comunicación instantáneo y cómodo de utilizar, a la vez que muy extendido, ha sido de gran ayuda para los estudiantes participantes del programa de bMentoría. Los encuentros presenciales se concentran en los días de tutoría en el centro asociado, son las sesiones más estructuradas y clarificadoras de dudas generales. La frecuencia con la que se han comunicado los estudiantes entre sí, ha variado a lo largo del curso, y se relaciona con momentos concretos; entrega de pruebas de evaluación continua (PEC), época de exámenes, resolución beca, etc. Es conveniente relacionar temas a tratar y medios de comunicación más pertinentes. Esta relación será un documento por entregar al bmentor cuando inicie la labor de bMentoría (Corral-Carrillo y Martin-Cuadrado, 2018).

Con respecto a la utilización de la plataforma de la UNED (aLF), nos encontramos con que no todos los bmentorizados están familiarizados/as con las TIC, y la ayuda de los bmentores es esencial e indispensable para que vayan avanzando y consigan en poco tiempo el manejo de la plataforma. Al mismo tiempo, se refuerza con las actividades que se realizan en las comunidades de acogida virtual para estudiantes nuevos, y con seminarios presenciales que se realizan en el centro asociado (Bodas y Corral-Carrillo, 2017). 
Los estudiantes nuevos que han participado activamente en el programa se sienten satisfechos de acuerdo con los resultados obtenidos con el programa, y en la mayoría de los casos han seguido un segundo año como bmentorizados.

De acuerdo con los resultados obtenidos, hemos de tener en cuenta que tenemos que pasar a una estructura de aprendizaje cooperativo-colaborativo, esto se traduce en calidad del proceso educativo. Se genera personalización de la enseñanza y estrategias mucho más amplias y sólidas que marcarán el futuro profesional, cumpliendo objetivos competenciales que le favorecerán en el futuro (Martín-Cuadrado et al, 2015). De este modo, los estudiantes bmentores podrán desarrollar competencias que va a redundar en una mejor preparación para su empleabilidad.

El Programa de Orientación Tutorial y bMentoría se enmarca en las metodologías de tutoría y ayuda entre iguales, que se han demostrado como especialmente apropiadas y aconsejables para la superación del sentimiento de aislamiento o soledad que en ocasiones puede aparecer en los estudiantes (Martín-Cuadrado y Corral-Carrillo, 2016).

Tenemos que adaptarnos a los nuevos caminos que se nos presentan en estos momentos en que los estudiantes son usuarios masivos de los diferentes medios de comunicación interpersonal, la teoría del desarrollo cultural nos acerca al conocimiento histórico-cultural del ser humano, éste es modelado por la cultura e interacciones sociales (Vygotsky, 2001).

Los Planes de Acogida se presentan en todas las Universidades. En este sentido añadir un programa de este tipo aportaría mayores oportunidades a los estudiantes nuevos, un igual que está con cada estudiante nuevo de manera individual, no desde la visión de déficit, sino desde la visión de "prevención", se trata de proveer de toda la ayuda, orientación acompañamiento, etc., para adaptarse a la Universidad y lograr superar con éxito las asignaturas (Wisker, Exley, Antoniou y Ridley, 2012).

La principal limitación de la presente investigación es la generalización que pueda hacerse de los resultados.

\section{Referencias bibliográficas}

Álvarez, P. y González, M. (2005). La tutoría entre iguales y la orientación universitaria. Una experiencia de formación académica y profesional. Revista Educar, 36, 107-128.

Arias, J. (2011). Aprendizaje mezclado (B-Learning) Modalidad de formación de profesionales. Revista Universidad EAFIT, 45(154), 70-77. http://www.um.es/ead/red/45/bolivar.pdf

Benavent, J. y Fossati, R. (1990). Un programa de compañero-tutor para los alumnos de Orientación Educativa de la Universidad de Valencia. Revista Española de Orientación Educativa y Vocacional. 66-80.

Bisquerra, R., y Álvarez, M. (1998). Modelos de orientación e intervención psicopedagógica. Barcelona: Praxis.

Bodas, E. y Corral-Carrillo, M. J. (2017). Talleres de orientación académica: estrategias vinculadas a fortalecer los procesos académicos de los estudiantes. En A.M. Martín-Cuadrado, E. Juan y N. Carriedo (coords.). Actas VIII Jornadas de Redes de Investigación en Innovación Docente (609-615). Madrid, España: UNED.

Cabero, J., Cebrián, M., Duarte, A., Gisbert, M., Martínez, F., y Salinas J. (2000). Las Nuevas Tecnologías para la mejora educativa. Sevilla: KRONOS. 
Calderón, M.C. y Corts, M.I. (1995). El método de enseñanza mutua. Su difusión en la América colonial española. Historia de la Educación, 14-15, 279-300

Callejo, J. (2001). Estudio de Cohorte de Estudiantes de la UNED: una Aproximación al Análisis del Abandono. Revista Iberoamericana de Educación a Distancia (RIED), (2), 33-69. https://doi.org/10.5944/ried.4.2.1179

Casado-Muñoz, R., Lezcano-Barbero, F. y Colomer-Feliu, J. (2015). Diez pasos clave en el desarrollo de un programa de mentoría universitaria para estudiantes de nuevo ingreso. Revista Electrónica Educare, 19(2), 155-180. https://doi.org/10.15359/ree.19-2.10

Castellano, F. y Delgado, L. (1996). Un programa de orientación universitaria: Participa. Revista de Educación de la Universidad de Granada.9, 65-78.

Corral-Carrillo, M.J. y Martín-Cuadrado, A.M. (2015). Mentoría en la universidad, nuevos modelos de comunicación e intercambio: Las Redes Sociales y la Comunidad de aprendizaje. En el marco del XX Congreso Internacional de Tecnologías para la Educación y el Conocimiento VII Congreso Pizarra Digital: Diversidad, Estrategias y Tecnologías: Tecnologías y Diversidad como base para la función docente. Madrid, España: UNED.

Corral-Carrillo, M.J. y Martín-Cuadrado A.M. (2017). La bMentoría como Experiencia Innovadora y formativa en el Centro Asociado de la UNED, en Sevilla. En A.M. Martín-Cuadrado, E. Juan y N. Carriedo (coords.). Actas VIII Jornadas de Redes de Investigación en Innovación Docente (609-615). Madrid, España: UNED.

Dewart, H., Drees, D., Hixenbaugh, P.,y Wiliams, D. (2004). Electronic peer mentoring: a scheme to enhance support and guidance and the student learning experience. Conference Psychology Learning and Teaching, Universidad de Strathclyde. Glasgow, UK.

Dubon, E., Climent, J. Pakhrou, T., Gil, A., Abad, L., y Martínez, J. (2011). La tutoría entre iguales como apoyo a los estudiantes de nuevo ingreso. $X$ Jornadas de redes de investigación en docencia universitaria. La participación y el compromiso de la comunidad universitaria.

Finkelsztein, D. (1994). Le monitorat: s'entraider pour réussir. Paris. Hachette

Fish, J. (2017). Aportes de la mentoría universitaria a la creación y el mantenimiento de una organización de aprendizaje continuo. Revista Gestión de la Innovación en Educación Superior, 2(2), 68-92.

Galino. A. (1968). Textos pedagógicos hispanoamericanos. Narcea.

García-Aretio, L. (1987). Rendimiento académico y abandono en la educación superior a distancia. Universidad Nacional de Educación a Distancia, Madrid: UNED.

García-Fuentes de la, C., y Manzano-Soto, N. (2015). El aprendizaje de los estudiantes de primero de carrera evaluado en ocho indicadores. Revista de Estudios e Investigación en Psicología y Educación, (01), 192-196. https://doi.org/10.17979/reipe.2015.0.01.1173

Homero (1991). La Odisea. Traducción Luis Segalá y Estalella. 24a edición. México: Editorial Porrúa.

Jarauta, B. (2014). El aprendizaje colaborativo en la universidad: Referentes y práctica. REDU. Revista de Docencia Universitaria, 12(4), 281-302. https://doi.org/10.4995/redu.2014.5624

Johnston, B. (2013). El primer año de universidad: una experiencia positiva de transición (Vol. 37). Narcea Ediciones.

Lancaster, J. (1803). Improvements in Education. London. Darton and Harvey.

Luque-Pulgar, E., García-Cedeño, F., y Santiago, C. de (2013). El abandono y el egreso en la UNED, en IUED. Actas de las VI Jornadas de Innovación Docente. (Madrid, UNED), 211-218. 
Manzano-Soto, N., Martín-Cuadrado, A. M., Sánchez M., Rísquez, A., Suárez M. (2012). El rol del mentor en un proceso de mentoría universitaria. Educación XX1, 93-118. https://doi.org/10.5944/educxx1.15.2.128

Manzano-Soto, N., y Roldán-Morales, C. (2015). Análisis de necesidades de orientación del estudiante de primer año en la Universidad Autónoma de Occidente. Revista Española de $\begin{array}{llll}\text { Orientación } y \text { Psicopedagogía } & \text { (REOP), 26(3), }\end{array}$ https://doi.org/10.5944/reop.vol.26.num.3.2015.16404

Martín-Cuadrado, A.M. (2011). Plan de Orientación Tutorial. C.A. de Talavera de la Reina. En A. Sánchez-Elvira Paniagua, A. y M. Santamaría Lancho (Coord.). (2011). Avances en la adaptación de la UNED al EEES. II Redes de Investigación en Innovación Docente. 34-55. Madrid, España: UNED.

Martín-Cuadrado, A. M. y Corral-Carrillo, M. J. (2016). La comunidad de aprendizaje y las redes en el Simposium auto-organizado B-Mentoria: orientación entre iguales en espacios mezclados (presenciales-tecnológicos) en el marco del 3o Congreso Internacional Corubric16, Tecnologías para la evaluación del Prácticum y las prácticas externas en contextos multidisciplinares. Universidad de Málaga 2016.

Miller, A. (2002). Mentoring students and young people: a handbook of effective practice. London: Kogan Page.

Moncada, L. F. (2014). La integración académica de los estudiantes universitarios como factor determinante del abandono de corto plazo. Un análisis en el sistema de educación superior a distancia del Ecuador. RIED. Revista Iberoamericana de Educación a Distancia, volumen 17, no 2, pp. 173-196. https://doi.org/10.5944/ried.17.2.12683

Moreno, M.L. (2003). La orientación profesional en los ámbitos académicos, en Sebastían, A. (coord. 2003). Orientación profesional. Un proceso a lo largo de la vida. Dykinson. Madrid. (pp.397-438)

RÍsquez, A. y Moore, S. (2005). Welcome to college? Transitional Adjustment and E-Mentoring. I Conference de Aimhigher, Mentoring in Higher Education, Stratford-upon-Avon, UK.

Rísquez, A. (2006). E-mentoría: avanzando la investigación, construyendo la disciplina. Revista complutense de educación, 17(2), 121.

Rísquez, A. (2010). La mentoría electronica entre pares para la adaptación a la universidad: Una experiencia de investigación aplicada. Tesis doctoral, Universidad Nacional de Educación a Distancia, Madrid.

Salinas, J. (2012). La investigación ante los desafíos de los escenarios de aprendizaje futuros. RED. Revista de Educación a Distancia, 32. 1-23. http://dx.doi.org/10.6018/red/50/13

Sánchez-Elvira, A., Fernández, E. y Amor, P. (2006). Self-regulated learning in distance education students: preliminary data. En A. Delle Fave (Ed.) Dimensions of well-being. Research and Intervention. Milano: Franco Angeli, 294-314

Santiago de, C. (2011). El abandono en primera matrícula en la UNED: Análisis comparado de la primera cohorte de Grados. Technical report, Unidad Técnica de Investigación del IUED, UNED.

Sánchez, M., Manzano, N., Suárez, M., Rísquez, A., Oliveros, L., Martín-Cuadrado, A., y Román, M. (2009). Sistema de orientación tutorial en la UNED: tutoría, mentoría y e-mentoría. Presentation, VI Jornadas Internacionales de Innovación Universitaria. Universidad Europea de Madrid.

Sánchez, M. F. (Coord.), Manzano, N., Suárez, M., Rísquez, A. y Oliveros, L., Martín, A. (Col.), Román, M. (Col.) y Vélaz, C. (Col.) (2009). Manual y Cuaderno de prácticas para el/la 
Compañero/a mentor/a. Programa de orientación tutorial y Mentoría en la UNED. Madrid: UNED.

Sánchez, M. F. (Coord.), Manzano, Martín, A., Oliveros, L., Rísquez, A. y Suárez, M., (2009). Desarrollo de un sistema de orientación tutorial en la UNED: primeros resultados del programa de mentoría. Revista de Mentoring \& Coaching 2: 39-55. https://doi.org/10.5944/educxx1.15.2.128

Sánchez, M.F, Manzano, N., Rísquez, A, y Suárez, M. (2011). Evaluación de un modelo de orientación tutorial y mentoría en la educación superior a distancia. Revista de Educación 356. 719-732. http://www.revistaeducacion.mec.es/doi/356 119.pdf

Sundli, L. (2007). Mentoring - A New Mantra for Education? Teaching and Teacher Education, 23, 201-214.

Valverde, A., García, E. y Romero, S. (2001). Desarrollo de un sistema de alumnos mentores en la Universidad de Sevilla. Aportación al Simposio de investigación sobre educación universitaria, dentro del X Congreso Nacional de Modelos de Investigación Educativa. Revista de Investigación Educativa, 19, 2, 626-627.

Valverde, A., Ruiz, C., García, E., Romero, S. (2003). Innovación en la orientación universitaria: la mentoría como respuesta. Contextos educativos: Revista de educación, (6), 87-112. http://dx.doi.org/10.18172/con.530

Vargas, M., González-Brignardello, M., Martín-Cuadrado, A.M. y Valero, E. (2014). El Plan de Acogida de Movilidad Internacional de la UNED - un Modelo de e-Mentoría. En A. Medina, C. Domínguez y M.L. Cacheiro (Coords.) Actas XIX Congreso Internacional de Tecnologías para la Educación y el Conocimiento y VI de Pizarra digital: diversidad, estrategias y tecnología. Diálogo entre Culturas. INTERESTRATIC. (CD).

Vega, M., (2000). Iniciativas para la integración: un programa premia la solidaridad entre estudiantes. Minusval. INMSERSO. 124

Vigotsky, L. (2001). Psicología Pedagógica. Buenos Aires: AIQUE.

Wisker, G., Exley, K., Antoniou, M. Y Ridley, P. (2012). Trabajando individualmente con cada estudiante. Tutoría personalizada, coaching, mentoring y supervisión en Educación Superior. Madrid: Narcea

Yusta, J., Cepero, M., Prieto, J., Abadía, A., y Bueno, C. (2015). Proyecto de mentoría en nuevos grados universitarios: la importancia de la organización. Experiencia de implantación del Programa Mentor en la Universidad de Zaragoza. Revista del Congrés Internacional de Docència Universitària i Innovació (CIDUI), (2).

Zimmerman, B. J. (1989). A social cognitive view of self-regulated academic learning. Journal of Educational Psychology, 81(3), 329-339

\section{Fuentes electrónicas}

Allueva, P. (2013). Mentoría en el Espacio Europeo de Educación Superior. En Á. Fidalgo y M. Sein-Echaluce (Eds.), II Congreso Internacional sobre Aprendizaje, Innovación y Competitividad (pp. 773-778). Madrid: Fundación General de la Universidad Politécnica de Madrid. (Documento electrónico). Recuperado de http://bit.ly/1coQUnZ 
Corral-Carrillo, M. J. y Martin-Cuadrado, A. M. (2018). Guía para la puesta en marcha de un programa de bMentoría en un centro asociado de la UNED. En IX Jornadas de Redes de investigación en innovación docente de la UNED. La profesionalización del docente a través de la innovación educativa. Madrid, España: UNED. [Audiovisual]. Madrid, España: CanalUNED. Recuperado de https://canal.uned.es/video/5a6f4903b1111f441b8b4582

Casado, R., Ruiz, M. (2009). Programa mentor: tutorías entre compañeros/as. En Actas del /I Congreso Internacional UNIVEST 09. (11.2009. Girona). (Documento electrónico). Recuperado de: http://bit.ly/1fjy0zp

Conferencia de ministros Europeos responsables de la educación superior (2009). El Proceso de Bolonia 2020. El Espacio Europeo de Educación Superior en la nueva década. Comunicado de Lovaina/Louvain-la-Neuve, Bélgica. 29 de abril. Consultado el 18 de abril, Lovaina/Louvainla-Neuve, 28-29 de abril de 2009. Recuperado de:

http://ec.europa.eu/europe2020/europe-2020-in-a-nutshell/targets/index es.htm

Instituto nacional de estadística (2016). Cifras INE [www.ine.es].

Martín-Cuadrado, A.M., CorraL-Carrillo, MJ., Márquez, M.D., González, F., García, C., Durán, P.J., Sánchez, M.J. y Molina, J.A. (2015). Serie BMentoría en Canal UNED. [Programa de Radio]. Madrid, España: CanalUNED. Recuperado de: https://canal.uned.es/serial/index/id/4271

Martín-Cuadrado, A.M. (2016). MOOC mis primeros pasos en la UNED. Guía para estudiantes nuevos. UNED. Madrid. Recuperado de: https://iedra.uned.es/courses/UNEDIUED/IUED DESCUBRE135/2016 T3/about

Plan de Orientación Universitaria Universidad de Zaragoza (POUZ). Recuperado de: http://www.unizar.es/ice/index.php/pouzalias

Plan TOURS Transición a la Universidad de Sevilla. Recuperado de: http://estudiantes.us.es/sites/default/files/ficheros/file/informacion/Plan\%20TOURS web v2.p $\underline{\mathrm{df}}$

Siemens, G. (2004). Conectivismo: una teoría del aprendizaje para la era digital. Publicado bajo Licencia Creative Commons 2.5. Recuperado de: http://edublogki.wikispaces.com/file/view/Conectivismo.

Servicio de promoción, información y orientación (SPIO) Universidad de Salamanca. Recuperado de: $\underline{\text { http://spio.usal.es/index.php?lang=es }}$

Fecha de entrada: 4 de septiembre de 2017

Fecha de revisión: 2 de mayo de 2018

Fecha de aceptación: 28 de diciembre de 2018 